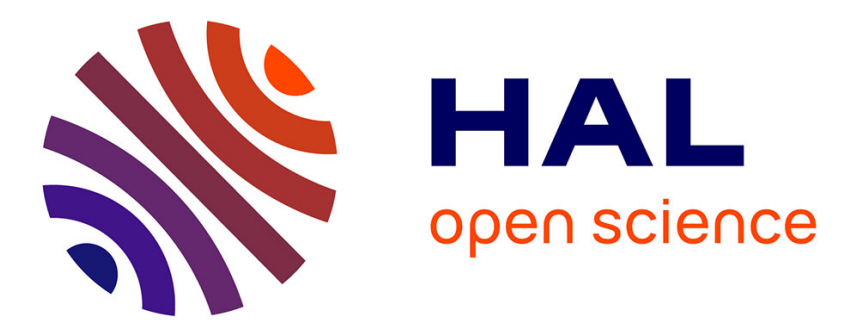

\title{
Mieux collecter les données personnelles sur Internet. Une étude qualitative auprès d'internautes français
}

Caroline Lancelot Miltgen, Jean-François Lemoine

\section{To cite this version:}

Caroline Lancelot Miltgen, Jean-François Lemoine. Mieux collecter les données personnelles sur Internet. Une étude qualitative auprès d'internautes français. Décisions Marketing, 2015, 79, pp.35-52. 10.7193/DM.079.35.52 . hal-02566835

\section{HAL Id: hal-02566835 \\ https://hal.science/hal-02566835}

Submitted on 19 Apr 2021

HAL is a multi-disciplinary open access archive for the deposit and dissemination of scientific research documents, whether they are published or not. The documents may come from teaching and research institutions in France or abroad, or from public or private research centers.
L'archive ouverte pluridisciplinaire HAL, est destinée au dépôt et à la diffusion de documents scientifiques de niveau recherche, publiés ou non, émanant des établissements d'enseignement et de recherche français ou étrangers, des laboratoires publics ou privés. 


\section{Mieux collecter les données personnelles sur Internet Une étude qualitative auprès d'internautes français.}

Caroline Lancelot Miltgen

Professeur Associée

Audencia Ecole de Management

clancelot@audencia.com

Jean-François Lemoine

Professeur des Universités

Université Paris 1 Panthéon Sorbonne (PRISM)

ESSCA Ecole de Management

jflemoine30@ hotmail.com

Published in

Décisions Marketing

No. 79 (Juillet-Septembre 2015), pp. 35-52

https://www.jstor.org/stable/44816102 


\title{
Mieux collecter les données personnelles sur Internet Une étude qualitative auprès d'internautes français.
}

\section{Résumé}

Dans un contexte de méfiance des internautes vis-à-vis de l'utilisation qui peut être faite des informations personnelles qu'ils fournissent sur Internet, quelles stratégies les entreprises peuvent-elles mettre en place afin de contourner leurs craintes, de les rassurer voire de les inciter à divulguer davantage leurs données? Afin d'apporter des éléments de réponse à cette question, une étude qualitative a été conduite auprès de trente internautes français. Elle permet de mettre à jour sept variables relatives au contexte de sollicitation (exposé de l'utilité et de la finalité de la demande, vocabulaire employé, tonalité de la sollicitation, quantité de données demandées, modalités de réponse proposées, possibilité offerte aux internautes de ne plus être sollicité ultérieurement) constituant autant de leviers d'action que les entreprises peuvent mobiliser dans leurs pratiques de collecte.

\section{Mots clés}

Données personnelles, vie privée, Internet, divulgation, contexte de sollicitation.

\section{Better collect Internet users' personal data A French qualitative study.}

\begin{abstract}
As Web users are more and more concerned regarding how their personal data are used, how can firms reduce their concerns, reassure them and even incite them to divulge more personal data? In order to answer this question, a qualitative study has been conducted with 30 French Internet users. This study underlines seven context variables (i.e. usefulness and aim of the data request, vocabulary used, request tone, quantity of requested data, responses modes, opportunity of not being asked next time) that represent useful strategies that firms could use when they collect personal data in order to bypass this concern.
\end{abstract}

\section{Keywords}

Personal data, privacy, Internet, disclosure, context of request. 


\section{Introduction}

De nos jours, les internautes sont régulièrement invités à communiquer leurs données personnelles que ce soit, par exemple, dans le cas d'une inscription en ligne à un club de fidélité, d'une participation à un jeu concours, d'une commande de catalogue ou de la création d'une page d'accueil personnalisée sur un site. Or, d'après une enquête réalisée par Havas Media en 2014, les consommateurs sont préoccupés par l'usage qui peut être fait des informations qu'ils laissent sur Internet. Plus exactement, 74\% des individus interrogés sont inquiets d'un éventuel usage frauduleux de leurs données, des risques de révélation d'informations privées et intimes (53\%) et de l'exploitation de leurs données à des fins de surveillance $(47 \%)^{1}$.

Dans un tel contexte de méfiance à l'égard d'Internet, comment les entreprises peuventelles contourner les réticences des internautes à divulguer leurs données personnelles et les amener au contraire à les diffuser sans crainte ? Apporter des éléments de réponse à ces interrogations est d'autant plus important pour les entreprises qu'elles sont soucieuses, d'une part, d'offrir à leurs clients des produits et/ou des services adaptés et, d'autre part, de mettre en œuvre à leur égard des actions de marketing relationnel efficaces.

Nous interroger sur les stratégies que les entreprises doivent mettre en œuvre pour faciliter la divulgation des données personnelles de leurs clients présente également un intérêt théorique. Cette étude vise ainsi à compléter la littérature existante consacrée à l'analyse et à la compréhension de la relation entre préoccupation pour le respect de la vie privée et dévoilement de données personnelles. Comme le soulignent en effet plusieurs auteurs (Lancelot Miltgen, 2008, Smith et al., 2011), il manque encore des recherches académiques susceptibles d'expliquer, dans un contexte de forte inquiétude pour le respect de la vie privée, ce qui amène réellement un internaute à accepter ou, au contraire, à refuser de diffuser ses données personnelles. La théorie du privacy calculus $^{2}$ permet d'analyser les comportements de dévoilement à l'aide du concept de valeur perçue de

\footnotetext{
${ }^{1}$ Havas Media (2014), Les Français et leurs datas. Disponible sur : http://www.havasmediaopendata.com/\#projectWrapper

${ }^{2}$ Mobilisée dans les travaux de Laufer et Wolfe (1977) et dans ceux de Stone et Stone (1990), cette théorie consiste à concevoir la vie privée non pas comme un droit à protéger mais comme une ressource à échanger. Cette vision économique de la vie privée considère que le comportement de l'individu est le résultat d'un arbitrage coûts/bénéfices.
} 
l'échange c'est-à-dire du différentiel perçu entre les bénéfices et les coûts associés au fait de divulguer des données personnelles (Dinev et Hart 2006). A ce jour, peu de travaux se sont penchés sur l'analyse précise des variables susceptibles d'influencer positivement ou négativement, d'après les consommateurs eux-mêmes, la valeur perçue de l'échange. Nous nous proposons donc, dans le cadre de cet article, de recenser les facteurs pouvant agir sur la valeur perçue de la divulgation de données personnelles en ligne et donc sur le comportement de dévoilement des internautes. A cette occasion, de nouvelles variables venant enrichir le cadre théorique du privacy calculus seront mises en évidence. A l'issue de cette contribution, nous serons donc en mesure de mieux rendre compte de la manière dont le consommateur évalue la valeur perçue de l'échange afin de savoir s'il envisage (ou non) de divulguer ses données personnelles.

Dans le but de renseigner les entreprises sur la manière dont il convient d'opérer pour collecter aisément les données personnelles des internautes, nous nous proposons de mener une étude empirique répondant aux trois questions suivantes :

- Quelles finalités les internautes associent-ils aux requêtes d'informations personnelles de la part des entreprises?

- Quels sont pour eux les principaux motifs de non divulgation des données personnelles?

- Sous quelles conditions les entreprises peuvent-elles les encourager à fournir de telles informations?

L'intérêt méthodologique de notre investigation est qu'elle repose exclusivement sur des éléments d'explication fournis par les internautes eux-mêmes et donc non préétablis par le chercheur. Traditionnellement peu prises en compte dans les recherches sur le comportement de dévoilement des individus, ces informations nous semblent devoir être privilégiées dans la mesure où elles sont susceptibles d'apporter un éclairage complémentaire à celui des responsables marketing en matière de compréhension des comportements de divulgation des données personnelles sur internet.

Après avoir exposé la notion de «données personnelles » et la manière dont les entreprises les collectent, nous nous intéressons au concept de préoccupation pour le respect de la vie privée et à ses conséquences sur le comportement des internautes. Nous présentons ensuite la méthodologie de recherche mise en œuvre lors de notre étude 
empirique. Puis, nos résultats sont exposés et des implications managériales quant à la façon dont les entreprises doivent effectuer les demandes d'informations personnelles en ligne sont dégagées.

\section{Collecte de données personnelles sur Internet et respect de la vie privée des internautes}

\section{Qu'est-ce qu'une donnée personnelle?}

Sur le plan juridique, la loi du 6 janvier 1978, relative à l'informatique, aux fichiers et aux libertés, considère comme une donnée à caractère personnel «toute information relative à une personne physique identifiée ou identifiable par référence à un numéro d'identification ou à un ou plusieurs éléments qui lui sont propres » (comme son nom, son numéro de sécurité sociale ou son adresse électronique) (article 2). Ainsi, d'un point de vue juridique, une donnée d'ordre sociodémographique collectée à travers un formulaire n'est pas personnelle en tant que telle mais le devient dès lors qu'elle est associée à des données permettant d'identifier clairement l'individu auquel elle est reliée (par exemple : numéro de client, nom, adresse email, etc.).

On peut classer les données personnelles en différents groupes : données d'identification (nom, adresse, numéro de sécurité sociale, etc.), caractéristiques sociodémographiques (sexe, âge, niveau d'études, etc.), données financières (niveau des revenus, montant des crédits contractés, etc.) et style de vie de la personne (informations qui décrivent les hobbies, les préférences, les valeurs et la personnalité de l'individu) (Gurau et al., 2003 ; O'Connor, 2003). On distingue aussi parfois les données relatives aux habitudes et aux comportements d'achat des consommateurs (montants des transactions réalisées, type de magasin fréquenté, etc.) (Phelps et al., 2000).

Pour le marketer, il est intéressant de distinguer non pas les données personnelles des données non personnelles mais plutôt d'identifier les données perçues comme sensibles par les consommateurs. De ce point de vue, les données diffèrent principalement par le niveau de risque auquel les individus s'exposent en les fournissant. Plus le risque est important plus les données sont considérées comme sensibles (Castaneda et al., 2007). Citons en exemple le cas des données financières, d’identification et, de manière générale, toutes les informations susceptibles de conduire à la réception d'offres commerciales 
(Ward et al., 2005).

\section{Comment l'entreprise collecte-t-elle les données personnelles des internautes?}

Les entreprises peuvent collecter les données personnelles des internautes de trois manières différentes :

- de manière directe : lorsque l'entreprise sollicite l'internaute par le biais d'un formulaire à remplir ;

- de manière indirecte : l'entreprise achète ou loue une base de données auprès de partenaires ou de sociétés spécialisées ou obtient des données déclaratives auprès d'autres clients (techniques de parrainage et de marketing viral) ;

- de manière discrète: des données essentiellement comportementales (comportements d'achat et de navigation notamment) sont collectées par le biais de techniques non directement décelables par l'internaute (techniques de tracking $^{3}$ et d'identification par le biais de cookies et de fichiers log).

Dans le cadre de cet article, nous nous intéressons essentiellement aux méthodes directes de collecte ${ }^{4}$.

\section{Préoccupation pour le respect de la vie privée et comportements des internautes.}

La communication de données personnelles est souvent considérée par les internautes comme une pratique à risque à l'égard de leur vie privée (Gauzente, 2003). Plus précisément, l'incertitude quant à la manière avec laquelle l'entreprise collecte et utilise ces informations constitue une des préoccupations majeures des consommateurs en matière de respect de leur sphère personnelle ${ }^{5}$. Une étude menée sur le sujet (Lusoli et Miltgen, 2009) montre en effet que les internautes français sont :

\footnotetext{
${ }^{3}$ Technique permettant de suivre le cheminement d'un internaute sur un site web depuis son arrivée jusqu'à la dernière page visitée.

${ }^{4}$ Dans la mesure où une partie de notre étude porte sur les motifs de non divulgation des données personnelles par les internautes (encadré 1), nous avons limité nos investigations aux méthodes directes de collecte puisque ce sont les seules qui supposent leur participation active (accepter de divulguer ou pas des informations). De plus, les critiques formulées par les internautes à l'égard des entreprises qui les contactent directement pour collecter leurs données personnelles sont tellement nombreuses et fréquentes qu'il nous a semblé important, dans un premier temps, de limiter nos investigations aux seules pratiques que les entreprises se doivent de mettre en œuvre en matière de collecte directe de données personnelles.

${ }^{5}$ Cette affirmation fait écho aux travaux de Solove (2006) qui mettent en évidence 4 catégories de problèmes liés à la vie privée dont ceux relatifs à la collecte et aux processus d'exploitation des données personnelles.
} 
- $\quad 80,3 \%$ à craindre que leurs données personnelles soient utilisées sans qu’ils en aient connaissance ;

- $\quad$ 80,1 \% à avoir peur que ces données soient transmises à des tiers sans leur accord ;

- $\quad 56,3 \%$ à penser que la divulgation des données personnelles risque de se traduire par la suite par l'envoi d'offres commerciales sans permission préalable

Cette enquête illustre les inquiétudes des internautes en matière de respect de leur vie privée. Par préoccupation pour le respect de la vie privée, il faut entendre «préoccupation qu'ont des individus, des groupes ou des institutions pour déterminer à quel point et de quelle manière l'information les concernant est communiquée aux autres ${ }^{6}$ (Westin, 1967). Cette préoccupation génère des comportements très différents, voire opposés, en matière de divulgation de données personnelles. Tandis que certains internautes affirment communiquer un nombre plus ou moins restreint d'informations (cas d'un niveau de préoccupation faible) (Wirtz, Lwin et Williams, 2007 ; Xu et al., 2011), d'autres préfèrent fournir volontairement des données mensongères (cas d'un niveau de préoccupation élevé) (Lancelot Miltgen, 2008). Cette difficulté à rendre compte du comportement des internautes en matière de dévoilement de soi est renforcée par l'existence d'un privacy paradox qui amène des individus hostiles à toute utilisation de leurs données personnelles à fournir pourtant un portrait extrêmement détaillé de leur profil et à le laisser libre d'accès à tout individu surfant sur le web. Pour rendre compte de ce paradoxe, il est fréquent de mobiliser la valeur perçue de l'échange c'est-à-dire l'arbitrage que l'internaute va opérer entre :

- les bénéfices associés à la divulgation des données personnelles (remise de cadeaux, bons d'achat ou autres contre parties commerciales) (Hui et al.,2007) ;

- les coûts résultant de cette opération (notamment la perception de vulnérabilité face à l'utilisation non maîtrisée des données fournies).

Dans le but de favoriser les comportements de dévoilement des internautes et de minimiser la communication de données erronées, les responsables de sites vont devoir, d'une part, identifier comment les consommateurs perçoivent les requêtes d'information

\footnotetext{
${ }^{6}$ Solove (2006) souligne l'extrême difficulté à définir clairement ce concept, d'où le problème, pour le législateur notamment, à identifier ce qui constitue une violation de ce droit et à guider les pratiques organisationnelles en matière de respect de la vie privée.
} 
et, d'autre part, mieux cerner leurs craintes en la matière. Afin de mettre en évidence ces éléments, une étude qualitative a été menée (encadré 1).

\section{Encadré 1 : Méthodologie de l'étude}

Notre étude qualitative repose sur 30 entretiens semi-directifs menés auprès d'internautes français. Ces derniers ont été choisis de manière à assurer, au sein de notre échantillon, une diversité de profils en matière de caractéristiques sociodémographiques (sexe et âge notamment) et de fréquence d'utilisation d'Internet (voir tableau suivant) car il a été démontré que ces variables influencent les perceptions et les comportements face à la collecte et à l'utilisation des données personnelles ( $\mathrm{Li}, 2011)$.

Composition de l'échantillon de répondants $(\mathrm{n}=30)$

\begin{tabular}{|l|c|}
\hline \multicolumn{1}{|c|}{ Caractéristiques des enquêtés } & Pourcentage \\
\hline Sexe & \\
Homme & $46,7 \%$ \\
Femme & $53,3 \%$ \\
\hline Age & \\
Moins de 25 ans & $26,6 \%$ \\
$25-34$ ans & $16,7 \%$ \\
$35-49$ ans & $30,0 \%$ \\
50 ans et plus & $26,7 \%$ \\
\hline Fréquence d'utilisation d'Internet & \\
Plusieurs fois par jour & $46,7 \%$ \\
Plusieurs fois par semaine & $33,3 \%$ \\
Plusieurs fois par mois & $20,0 \%$ \\
\hline
\end{tabular}

Les entretiens avaient une durée moyenne de 80 minutes. Ils ont été enregistrés et intégralement retranscrits. Les thèmes abordés étaient, entre autres, les suivants : les perceptions des finalités de la collecte de données personnelles, les motifs de non divulgation de données personnelles en ligne. Les informations obtenues auprès des internautes ont fait l'objet d'une analyse de contenu et, plus précisément, d'une analyse thématique. A cette occasion, les pourcentages de citation (fréquences d'occurrence) des différents thèmes abordés ont été calculés. En raison du caractère qualitatif de notre recherche, ces indicateurs quantitatifs ne prétendent pas à une quelconque représentativité statistique mais se proposent de synthétiser, sous forme chiffrée, l'information collectée.

\section{Perceptions des finalités de la collecte de données personnelles}


Il ressort de notre étude qualitative que les répondants font preuve d'une très grande lucidité quant aux objectifs poursuivis par les entreprises quand celles-ci collectent des données auprès de leurs prospects et/ou de leurs clients. Ils distinguent très nettement les trois finalités suivantes :

- mieux connaître les clients pour mieux les démarcher (49\% des citations) ;

- faciliter la conception d'une nouvelle offre commerciale (33\% des citations)

- permettre la constitution de fichiers clients (18\% des citations).

\section{Mieux connaître les clients pour mieux les démarcher}

La finalité commerciale de la collecte de données personnelles n'échappe pas aux répondants qui y voient avant tout une opération permettant de mieux connaître le consommateur :

"Ils font ça pour mieux cerner le client...mieux connaître son pouvoir d'achat » (Homme, 52 ans, plusieurs fois par jour);

"Tout ça c'est pour savoir ce que les gens achètent ou pour savoir ce qui est susceptible de les intéresser » (Femme, 55 ans, plusieurs fois par mois) ;

"C'est l'occasion pour eux de comprendre ce que nous consommons...de découvrir ce que nous aimons et ce dont nous avons besoin » (Homme, 20 ans, plusieurs fois par jour) ;

"Ils veulent définir mon profil de consommateur, mes potentialités en matière économique, mes centres d'intérêts, ce que je peux me permettre...à la limite ce que je ne peux pas me permettre » (Femme, 40 ans, plusieurs fois par semaine).

Cette première finalité semble plutôt perçue positivement dans la mesure où les consommateurs interrogés y voient une prise en compte valorisante de leurs spécificités.

Les actions qui consistent ensuite à démarcher les clients sur la base des informations collectées ne leur sont pas non plus étrangères ...

"On nous demande tout ça pour nous relancer après...c'est très commercial » (Homme, 33 ans, plusieurs fois par semaine);

«Je suis pas naïve, je sais bien que je vais recevoir plein de pubs après...des petits mails sournois pour essayer de me vendre leurs produits »(Femme, 45 ans, plusieurs fois par semaine) ; 
"Tout ça c'est commercial, leur but c'est quand même de nous noyer sous la pub après » (Homme, 28 ans, plusieurs fois par jour).

... mais elles semblent cette fois-ci évaluées plus négativement, le vocabulaire employé (« sournois », « noyer ») indiquant clairement les limites associées à ce type de pratiques.

\section{Faciliter la conception d'une nouvelle offre commerciale}

Pour beaucoup de répondants, la collecte de données personnelles constitue également pour les entreprises un outil d'aide à la décision en matière d'amélioration de l'offre existante et de conception et/ou de lancement de nouveaux produits :

"Tout ce qu'on me demande, ça peut les aider à faire évoluer leurs produits...ça peut apporter un plus au niveau de la qualité...avec ces informations, il y a des gens qui vont être plus performants »(Femme, 37 ans, plusieurs fois par jour) ;

«Je pense que les entreprises utilisent ces données pour améliorer leurs produits et pour en sortir des nouveaux....ça leur apporte plein de choses tous ces sondages, toutes ces recherches »(Femme, 60 ans, plusieurs fois par mois).

Si certains consommateurs reconnaissent que cela leur profite indirectement (« un plus au niveau de la qualité »), d'autres soulignent surtout les avantages que cela procure aux entreprises («ça leur apporte plein de choses ») suggérant ainsi une possible perception d'iniquité dans la répartition des bénéfices.

\section{Permettre la constitution de fichiers clients}

Les répondants associent aussi les opérations de demande d'informations personnelles à un moyen pour l'entreprise de se constituer aisément des fichiers clients qu'elles pourront ensuite exploiter à leur guise :

"Quand je leur communique toutes ces données, je sais que ça améliore considérablement leur fichier client et qu'ils auront deux ou trois fois plus de chance de faire mouche quand ils me proposeront un nouveau produit» (Homme, 22 ans, plusieurs fois par jour) ;

"Tout ça c'est pour revendre des fichiers...si je leur dis que je gagne correctement ma vie, que je suis un passionné d'informatique, c'est sûr qu'ils peuvent vendre leur fichier à des sociétés qui vendent de l'informatique » (Homme, 24 ans, plusieurs fois par jour) ;

«Je pense que toutes ces demandes, c'est pour refourguer ça à d'autres entreprises de toute façon » (Femme, 47 ans, plusieurs fois par semaine).

D'après le vocabulaire employé, il apparaît clairement que l'exploitation interne des données (par l'entreprise qui les a récoltées) est davantage tolérée (« deux ou trois fois plus de chance de faire mouche ») que l'exploitation externe (après transfert des données 
à d'autres entreprises), cette dernière amenant alors les consommateurs à se montrer soit résignés («c'est sûr qu'ils peuvent vendre leur fichier») soit démunis (« de toute façon »).

L'intérêt de cette première série de résultats est qu'elle nous renseigne sur ce que les internautes comprennent de la finalité des opérations de collecte des données personnelles de la part des entreprises. Il ressort de nos analyses que les consommateurs ne sont pas dupes quant aux avantages que les entreprises retirent de telles actions. Une certaine frustration transparait toutefois des discours, semblant indiquer que les entreprises auraient tout intérêt à mettre davantage l'accent sur les avantages que les consommateurs peuvent eux-mêmes en retirer (personnalisation des offres, participation et coconstruction des nouveaux produits, amélioration de la qualité des produits, etc.). En s'engageant de manière plus transparente sur les données dont elles disposent déjà, celles qu'il leur faudrait obtenir et l'utilisation qu'elles en font, les entreprises pourraient accroitre la confiance de leurs clients. Par ailleurs, permettre au consommateur de bénéficier en retour des avantages qu'elles-mêmes retirent de la collecte des données personnelles pourrait être une source de compétitivité payante sur le long terme. D'une manière générale, tous ces éléments relèvent de la nécessité d'une plus grande autonomie des individus dans leurs relations aux organisations ${ }^{7}$. Comme le résume Kaplan (2010), quelque chose d'essentiel a changé : les individus disposent désormais de plusieurs moyens de traitement et d'échange d'informations. Ils s'en servent pour s'affirmer, s'exprimer, échanger, collaborer, contracter, apprendre, etc. A l'ère du big data, l'enjeu du customer empowerment semble donc crucial.

\section{Motifs de non divulgation de données personnelles à une entreprise}

Quatre grands motifs de non divulgation des données personnelles ressortent du discours des répondants. Ils concernent tour à tour :

- le caractère trop intime des données collectées (33\% des citations) ;

- la peur d'apparaître dans des fichiers (27\% des citations) ;

- la non-maîtrise du processus de traitement des données (20\% des citations) ;

\footnotetext{
${ }^{7}$ Nous reprenons ici quelques-unes des idées défendues notamment par la FING (Fondation Internet Nouvelle Génération)

(http://doc.openfing.org/CONFIANCE/ConfianceNumerique_SyntheseFinale_Fevrier2011.pdf).
} 
- les craintes liées à l'exploitation des données collectées (20\% des citations).

\section{Le caractère trop intime des données collectées}

La première raison pour laquelle les répondants peuvent être hostiles à la divulgation de leurs données personnelles est liée à leur nature. Si les informations demandées sont jugées trop personnelles, trop sensibles, trop intimes ou trop liées à leur vie privée, les consommateurs risquent de ne pas les communiquer :

"Si on me demande des choses très générales, ça ne me dérange pas...mais si on commence à m'interroger sur des choses plus pointues, plus précises, là ça va me poser des problèmes » (Femme, 39 ans, plusieurs fois par semaine) ;

"S'il y a des questions qui me semblent aller trop dans la sphère de la vie privée, je ne réponds pas » (Homme, 28 ans, plusieurs fois par jour) ;

"Dans les questions qui sont formulées parfois, je trouve que c'est trop intime et que c'est pousser le bouchon un peu loin » (Femme, 64 ans, plusieurs fois par mois) ;

"J'ai pas envie d'une intrusion agressive dans ma vie privée, style il faut acheter ça parce que vous êtes comme ça» (Homme, 47 ans, plusieurs fois par mois).

Si la distinction entre personnel, intime, sensible ou lié à la vie privée existe, elle reste assez floue pour les consommateurs qui semblent surtout distinguer ce qu'ils estiment pouvoir être dit à une entreprise (« des choses très générales ») et ce qui s'avère relever du domaine privé.

\section{La peur d'apparaître dans des fichiers}

La crainte d'être catégorisé dans des fichiers d'entreprises constitue un deuxième obstacle important à la divulgation d'informations personnelles :

"L'idée de se retrouver dans un fichier ultra détaillé, ça ne me plaît pas particulièrement »(Femme, 24 ans, plusieurs fois par jour);

"On sait que l'on va être fiché alors le but du jeu c'est de l'être le moins possible en ne racontant pas tout ou en disant parfois n'importe quoi » (Homme, 31 ans, plusieurs fois par semaine) ;

"Evidemment on rentre après dans des cases...la ménagère de moins de 50 ans, les jeunes entre 15 et 35 ans...tout ça ne me plaît pas trop alors je freine un peu des quatre fers » (Femme, 48 ans, plusieurs fois par jour);

"Je réponds pas, ça m'ennuie parce qu'après tu rentres dans une catégorie et on te lâche plus » (Homme, 55 ans, plusieurs fois par semaine).

Cette catégorisation qui correspond à une démarche classique de segmentation en marketing est perçue négativement par les consommateurs qui semblent dénoncer le côté 
réducteur et persistant de cette approche. En réaction à cette dernière, les consommateurs mettent manifestement en œuvre des stratégies de contournement (« en ne racontant pas tout ») ou de travestissement de la réalité (« en disant parfois n'importe quoi ») pour éviter de trop se dévoiler.

Au-delà du simple fait d'apparaître dans un fichier client à caractère commercial, c'est aussi la symbolique de l'enregistrement d'individus dans une base de données qui gêne particulièrement les répondants :

"Quoi que tu fasses tu es cerné, Big Brother est partout » (Homme, 22 ans, plusieurs fois par jour) ;

"Ça fait un peu Big Brother, ils savent où on va, ce qu'on fait, ce qu'on aime » (Homme, 30 ans, plusieurs fois par semaine) ;

"Historiquement les listes, ça a toujours une connotation...quand même pas trop libérale...que ce soit les listes noires d'Hollywood au temps du McCartisme...les fichiers, c'est généralement pas bon signe »(Femme, 55 ans, plusieurs fois par mois).

Si le côté historique des listes semble inquiéter («c'est généralement pas bon signe »), les consommateurs interrogés paraissent assez fatalistes («quoi que tu fasses ») quant à la démarche de surveillance qui s'est engagée avec l'apparition des TIC. Par ailleurs, alors que 'Big Brother' faisait originellement référence au côté étatique de la surveillance, les consommateurs assimilent aujourd'hui davantage ce personnage aux entreprises qui cherchent à en savoir le plus possible sur leurs clients et prospects quitte à se montrer parfois très intrusives.

\section{La non-maîtrise du processus de traitement des données}

Le fait de ne pas maîtriser le processus de traitement des données fournies inquiète également les répondants. Ces derniers hésitent donc souvent à divulguer des informations personnelles car ils ne savent pas réellement à qui ils les communiquent :

"La question que je me pose quand on me demande ces renseignements, c'est où estce que ça va aller ce que je donne? »(Femme, 19 ans, plusieurs fois par jour) ;

"Internet, c'est une grosse nébuleuse, on ne sait pas où passent nos données" (Homme, 52 ans, plusieurs fois par jour) ; 
"Ça ne me gêne pas de donner des informations personnelles quand je connais la personne... si la FNAC me demande des renseignements, je le fais car la FNAC je sais qui c'est mais sur Internet, c'est pas toujours le cas et là ça me dérange » (Homme, 57 ans, plusieurs fois par jour).

Davantage de clarté et de contrôle concernant les personnes (physiques et morales) amenées à collecter, traiter, stocker, utiliser ce type de données rassurerait probablement un grand nombre de consommateurs qui identifieraient alors un interlocuteur à qui s'adresser en cas de questions à poser ou de problèmes à résoudre.

\section{Les craintes liées à l'exploitation des données collectées}

La crainte que les données fournies soient divulguées et exploitées sans le consentement préalable et systématique du répondant justifie souvent leur non communication :

«Si tu fournis des renseignements, tu peux ensuite recevoir des messages de n'importe qui que tu ne connais pas....et tu ne sais même pas comment ils ont eu ton adresse " (Femme, 42 ans, plusieurs fois par semaine) ;

"C'est ennuyeux qu'ils utilisent le nom de toutes les personnes du fichier sans demander l'autorisation » (Homme, 18 ans, plusieurs fois par jour) ;

"Je ne donne plus d'informations car après ils t'envoient des échantillons alors que tu n'as rien demandé et ils persistent à t'envoyer cela toutes les semaines » (Homme, 33 ans, plusieurs fois par semaine).

Cette demande de consentement préalable va dans le sens de la LCEN (loi pour la confiance dans l'économie numérique) du 21 juin 2004 qui indique que les consommateurs doivent donner leur consentement pour tout programme de nature commerciale visant à exploiter leurs données personnelles. Par ailleurs, nos analyses semblent confirmer la crainte des consommateurs de voir leurs données exploitées sans qu'ils en aient été informés au préalable.

Il semblerait, par ailleurs, que cette demande de consentement préalable formulée par les consommateurs ne relève d'une posture ni automatique ni systématique mais plutôt d'une réaction liée à une expérience négative dans ce domaine («je ne donne plus »). La confiance étant brisée, un cercle vicieux peut alors s'engager non seulement vis-à-vis de l'entreprise à l'origine de la divulgation mais aussi, potentiellement, vis-à-vis de l'ensemble des organisations à vocation commerciale, les consommateurs pouvant alors vivre chaque démarche de marketing direct (relance commerciale) comme une agression («alors que tu n'as rien demandé »). 
D’une manière générale, ce que les entreprises peuvent faire des données transmises inquiètent les répondants. Deux types de risques sont recensés. Le premier concerne les opérations d'échange et/ou de vente de bases de données que les firmes peuvent réaliser entre elles :

"Ils s'échangent des fichiers entre eux, c'est connu et ça ne me plaît pas » (Femme, 47 ans, plusieurs fois par semaine) ;

"J'hésite à communiquer des informations personnelles car je crains qu'elles soient ensuite transmises à des tiers » (Homme, 52 ans, plusieurs fois par jour) ;

"J'aime pas imaginer que les renseignements que je fournis puissent être donnés ensuite à des gens que je connais pas » (Femme, 19 ans, plusieurs fois par jour).

Le second risque a trait au démarchage commercial dont les consommateurs peuvent faire l'objet suite à la divulgation de leurs données personnelles :

«Je me dis que si je renvoie le questionnaire, je vais être assaillie de toute part et ça va être plus des ennuis qu'autre chose » (Femme, 42 ans, plusieurs fois par semaine) ;

"Je ne donne pas d'informations car je risque de recevoir sans arrêt du courrier, des coups de téléphone, des choses comme ça...s'ils me demandent ces informations c'est qu'ils vont s'en servir » (Homme, 65 ans, plusieurs fois par mois) ;

«Le risque, pour moi, c'est d'être bombardée de publicités dans ma boîte aux lettres parce que j'ai répondu à un questionnaire...et je me dis oh la la, je n'aurais jamais dû y répondre... maintenant je ne peux plus $m$ 'en sortir, ils m'envoient de la documentation tout le temps »(Femme, 52 ans, plusieurs fois par semaine).

Si le premier risque donne lieu à des propos plutôt modérés, le second semble occasionner des réactions plus virulentes, certains consommateurs apparaissant visiblement excédés par ces pratiques de démarchage à la lecture du vocabulaire employé pour les décrire (« être assaillie », « sans arrêt », « être bombardée »).

Sur les quatre motifs de non divulgation de données personnelles mentionnés par nos répondants, trois ont déjà été mis à jour dans la littérature. Il s'agit du caractère trop intime des données collectées, de la non maîtrise du processus de traitement des informations et des craintes liées à l'exploitation des données collectées. Ils font simultanément référence aux notions de sensibilité des données personnelles, de contrôle (ou d'absence de contrôle) sur le traitement des informations fournies et de confidentialité de ces dernières. En revanche, la peur d'apparaître dans les fichiers est une crainte qu'on ne retrouve pas dans la littérature traditionnelle sur le comportement de dévoilement des internautes. Elle fait écho au degré de vulnérabilité que peut éprouver l'internaute face aux pratiques de catégorisation des clients auxquelles les entreprises se livrent sur la base des données 
collectées. Au-delà de l'enrichissement de la littérature en matière de motifs de non divulgation des données personnelles (Lancelot Miltgen 2008 ; Phelps et al. 2000), la mise à jour de cette crainte supplémentaire s'avère également utile pour les entreprises qui, pour tenter de la contourner, devront faire preuve de pédagogie dans leur pratique de sollicitation des internautes (en leur expliquant, par exemple, l'utilité et la finalité des classifications clients opérées sur la base des données que ces derniers ont bien voulu communiquer) ${ }^{8}$.

\section{Stratégies recommandées pour encourager la divulgation d'informations personnelles}

A partir de l'analyse des entretiens, quatre types de recommandations peuvent être adressés aux entreprises soucieuses de collecter des informations personnelles auprès de leurs clients et des consommateurs en général. Elles ont pour objectif majeur de contourner les quatre motifs de non divulgation évoqués par nos répondants.

\section{- Offrir une contrepartie aux internautes}

D’une manière générale, les individus divulguent rarement gratuitement leurs données personnelles :

«Si je donne des informations, c'est pour avoir un retour en général » (Femme, 55 ans, plusieurs fois par mois) ;

«Je ne fournis des renseignements que si l'entreprise m'offre quelque chose...c'est du donnant-donnant » (Homme, 34 ans, plusieurs fois par semaine) ;

"Si ces informations vont me permettre d'avoir quelque chose en plus de l'entreprise, je vais les donner » (Homme, 18 ans, plusieurs fois par jour).

Ils attendent donc de l'entreprise de recevoir en échange de leurs données personnelles des services ou des offres commerciales attrayantes :

«Si je communique des renseignements, j'aime bien recevoir des services gratuits...c'est un échange de bons procédés » (Homme, 47 ans, plusieurs fois par mois) ;

« Je suis étudiante et si je réponds, ça me fait $20 \%$ de réduction...là ça ne me dérange pas de donner car il y a des promos » (Femme, 19 ans, plusieurs fois par jour) ;

\footnotetext{
${ }^{8}$ La section suivante reviendra plus en détail sur la manière dont les entreprises devront faire preuve de pédagogie vis-à-vis des internautes dans la mesure où il s'agit d'une des quatre stratégies que nous avons identifiées afin de favoriser la divulgation de leurs données personnelles.
} 
"C'est vrai que je suis tentée de leur donner des infos car ils vont m'envoyer des bons d'achat, de réduction...je me dis tiens quels sont les produits qu'ils peuvent me proposer $\gg$ (Femme, 55 ans, plusieurs fois par mois).

Malgré les craintes recensées précédemment, les internautes sont donc prêts à dévoiler des informations personnelles sous réserve d'obtenir une contrepartie (« échange de bons procédés ») jugée satisfaisante (« là ça ne me dérange pas de donner car il y a des promos »). En ce sens, leur comportement relève d'un arbitrage coût/bénéfice classiquement pratiqué par les consommateurs (privacy calculus) (Xu et al., 2011) mais qui s'avère d'une acuité accrue lorsque le dévoilement de données personnelles s'opère en ligne. Internet accentue en effet les risques auxquels les individus sont exposés en ce qui concerne leur vie privée et une contrepartie satisfaisante doit donc être offerte pour compenser ce risque (Paine et al., 2007).

De manière pragmatique, les entreprises doivent donc réfléchir aux types de contreparties à offrir à leurs visiteurs en échange de leurs données, et ce, en fonction de l'appétence de ces derniers pour tel ou tel type de bénéfice. Il conviendra alors, dans la mesure du possible, d'adapter les avantages proposés au profil du visiteur et à l'historique de la relation.

\section{- Faire preuve de pédagogie dans la présentation de la demande}

De manière à contourner les résistances des consommateurs liées à la divulgation et à l'utilisation ultérieure possible des données fournies, les entreprises se doivent de faire preuve d'une très grande clarté tant sur l'utilité de la demande effectuée...

«Si je sens que cette information là, elle leur est nécessaire, je vais la donner » (Homme, 20 ans, plusieurs fois par jour) ;

«Je le ferai parce que j'en vois l'utilité » (Homme, 47 ans, plusieurs fois par mois).

... que sur sa finalité.

«Si je sais que ça va pas m'importuner » (Femme, 64 ans, plusieurs fois par mois) ;

"Si j'ai l'impression que ça sert à rien, c'est sûr que j'ai pas envie de le faire...si je vois pas le but derrière » (Homme, 30 ans, plusieurs fois par semaine) ;

«Il faut qu'il m'explique pourquoi il veut mon adresse personnelle » (Femme, 48 ans, plusieurs fois par jour);

«Si c'est pour de la publicité, je ne donne pas (Homme, 57 ans, plusieurs fois par jour) ; 
«Si ça reste dans des fichiers commerciaux...c'est pas trop dramatique...ça pourrait avoir des fins politiques et là c'est quand même un peu gênant» (Homme, 28 ans, plusieurs fois par jour) ;

«J'accepte dès l'instant que c'est juste pour t'interroger, t'envoyer de la documentation $\gg$ (Femme, 52 ans, plusieurs fois par semaine).

Ce souci de pédagogie peut permettre d'atténuer le sentiment de non maîtrise du processus de traitement des données personnelles tel qu'évoqué par nos répondants. En renseignant les internautes sur la finalité véritable de la collecte d'informations, les entreprises peuvent espérer (cf. partie sur les finalités perçues de la collecte) :

- limiter les perceptions négatives associées aux pratiques de démarchage et de constitution de fichiers clients ;

- renforcer l'idée selon laquelle la divulgation de données peut être une façon de participer à la création et à l'amélioration de l'offre de l'entreprise.

\section{- Soigner la formulation de la demande}

Pour accroître la probabilité de réponse des consommateurs, il ressort de nos entretiens que la requête de l'entreprise doit également présenter les caractéristiques suivantes :

1. Elle ne doit porter que sur un nombre limité d'informations jugées non intrusives dans la vie privée des répondants :

«Je donne d'autant plus que je ne dois divulguer que le minimum» (Femme, 24 ans, plusieurs fois par jour);

«Je ne fournis que les informations qui permettent de valider le formulaire... tout ce qui est facultatif, je le mets pas » (Homme, 28 ans, plusieurs fois par jour) ;

« Je donne que ce qui est indispensable... tant que ça se limite à une certaine sphère... ça ne me dérange pas dès l'instant qu'il n'y a pas de questions trop privées » (Femme, 52 ans, plusieurs fois par semaine).

Il résulte de cette condition que le formulaire de données doit être limité à un faible nombre de champs à compléter, ces champs devant également correspondre à des données générales et peu sensibles. En pratiquant de la sorte, l'entreprise peut espérer réduire la crainte d'une trop forte intrusion dans la vie privée des consommateurs.

2. Elle doit recourir à un vocabulaire compréhensible par tous et jugé non agressif par la cible visée : 
«Tout dépend de la façon dont les questions sont tournées...tout dépend du ton, des mots employés, s'il y a des mots que je ne comprends pas, je vais très mal le prendre » (Femme, 39 ans, plusieurs fois par semaine) ;

"Quand je trouve que c'est un peu trop agressif...s'ils veulent un fichier ils l'auront...il y aura que des conneries » (Homme, 28 ans, plusieurs fois par jour) ;

«Je le ferai peut être un jour si on est moins agressif dans les demandes » (Homme, 52 ans, plusieurs fois par jour);

«S'ils insistent trop, c'est plus la peine, je réponds plus » (Femme, 29 ans, plusieurs fois par jour).

Ce choix du vocabulaire peut permettre de minimiser les stratégies utilisées par les internautes pour éviter de trop se dévoiler et notamment celles qui consistent à fournir des données erronées (Lwin et Williams, 2003).

3. Elle doit offrir aux répondants des alternatives tant en matière de modalités de réponses fournies...

« Je donne d'autant plus d'infos que j'ai le choix dans les réponses, que c'est totalement libre (Femme, 39 ans, plusieurs fois par semaine) ;

«Selon que c'est une question ouverte ou fermée, j'ai pas la même envie de répondre » (Homme, 20 ans, plusieurs fois par jour).

... que de possibilités de ne plus être sollicité ultérieurement.

«À la fin des demandes, il y a de plus en plus souvent la petite case à cocher pour ne plus être dérangé...j'insiste là-dessus mais je pense que c'est important d'avoir le choix ... avec cette mention, on a le choix» (Homme, 34 ans, plusieurs fois par semaine) ;

"Le fait de pouvoir cocher ou décocher fait qu'on prend un peu plus conscience de ce qu'on fait » (Femme, 29 ans, plusieurs fois par jour).

Ces verbatim confirment la nécessité pour les entreprises d'offrir aux internautes une grande liberté de réponse et la possibilité d'exprimer leur consentement quant à l'utilisation ultérieure des données. De telles pratiques sont l'occasion de réduire les craintes formulées par les répondants quant à l'exploitation de leurs données personnelles. Elles pallient également l'absence fréquente d'autorisation de transmission des informations à des tiers (Gauzente, 2003).

\section{- Gagner la confiance du répondant}


Pour gagner la confiance des internautes, il convient de leur fournir toutes les informations qu'ils jugent utiles pour apprécier la crédibilité, d'une part, de l'entreprise demandeuse d'informations, d'autre part, de la requête à laquelle elle se livre.

Pour y parvenir, il convient de renseigner précisément les répondants sur :

1. L'identité de l'interlocuteur. Cela peut passer notamment, au regard de nos entretiens, par la présentation de données relatives au nom, à la taille et à la réputation de l'entreprise :

«Évidemment, tout dépend de la boîte qui me demande ça, si c'est pas trop connu, je serai peut-être un peu plus réticent...le nom, si je connais ou si je connais pas...le bouche à oreille, ça y fait beaucoup » (Homme, 52 ans, plusieurs fois par jour) ;

"Si j'arrive pas à connaître l'identité de mon interlocuteur, c'est clair je ne donne rien...j'aime bien connaître celui qui va utiliser mes informations» (Femme, 55 ans, plusieurs fois par mois) ;

«Je crois que l'aspect grand groupe c'est sécurisant en fait...parce qu'on sait qu'on aura des moyens faciles si jamais il y a un problème...on se dit qu'ils feront un usage réglo de nos données et que si une erreur arrive, elle pourra être rectifiée plus facilement » (Femme, 52 ans, plusieurs fois par semaine) ;

"Tout dépend de l'aura qu'a l'entreprise...enfin à mes yeux...comment je vois l'entreprise...l'aura de sérieux qu'elle dégage à travers son site »(Homme, 34 ans, plusieurs fois par semaine).

2. Le caractère confidentiel du dévoilement. Les internautes déclarent divulguer d'autant plus leurs données personnelles qu'il est précisé, lors de la requête, que leur anonymat sera respecté.

« Je donne si ça reste anonyme »(Homme, 28 ans, plusieurs fois par jour) ;

«Je suis prête à fournir des informations s'il est expliqué quelque part qu'elles vont être utilisées de manière anonyme » (Femme, 55 ans, plusieurs fois par mois).

3. L'aspect sécurisé du site. Il ressort de nos entretiens que la transmission d'informations sur la sécurisation du site est un préalable nécessaire à la divulgation de données personnelles.

«Ça ne me gêne pas de donner des renseignements sur moi si je sais que tout cela transite sur des sites sécurisés» (Homme, 20 ans, plusieurs fois par jour);

«À partir du moment où je vois le petit logo sécurisé, je peux fournir des données personnelles sans problème » (Femme, 18 ans, plusieurs fois par jour). 
Ces verbatim confirment ainsi l'importance de la réputation et de la sécurisation du site dans l'acceptation de dévoiler des données personnelles en ligne. Cette dernière semble également expliquée par un autre déterminant moins souvent étudié dans les études en marketing, à savoir la perception de la confidentialité du dévoilement.

Parmi les quatre stratégies mises à jour, deux ont déjà été étudiées dans la littérature. Il s'agit de celle qui consiste à offrir une contrepartie aux internautes et de celle qui vise à gagner leur confiance. Si la première se propose d'accroître les bénéfices que l'individu peut retirer d'une divulgation de ses données personnelles, la seconde s'attache plutôt à réduire les coûts et les craintes liées à la communication d'informations. Les stratégies visant à faire preuve de pédagogie dans la présentation de la demande et à soigner la formulation de la requête sont, en revanche, nouvelles dans la littérature dédiée au comportement de dévoilement des internautes. Elles mettent en avant l'importance des caractéristiques du contexte de sollicitation du consommateur assimilables aux facteurs situationnels couramment mobilisés dans la recherche en marketing et définis comme « l'ensemble des facteurs particuliers à un lieu et une période d'observation qui ne découle pas de connaissances personnelles (intra-individuelles) ou de réactions face à un stimulus et qui a un effet systématique et démontrable sur le comportement habituel du consommateur » (Lemoine, 2001a, 2001b). Si le rôle du contexte ${ }^{9}$ de sollicitation dans la divulgation des données personnelles a déjà été mis à jour dans la littérature (e.g. Lancelot Miltgen, 2008, 2010), il n'a été abordé, jusqu'à présent, qu'au travers du type de données demandées (degré de sensibilité des informations) et des caractéristiques de l'interlocuteur demandant les données (sa notoriété) (Lancelot Miltgen, 2010). Or, nos résultats tendent à prouver que sept nouvelles dimensions du contexte de sollicitation des internautes peuvent être prises en compte par les entreprises lors de l'élaboration de leurs pratiques de collecte de données. Les deux premières concernent la présentation de l'objectif de la sollicitation (exposé de l'utilité et de la finalité de la demande) et les cinq suivantes la manière dont la sollicitation doit être formulée (quantité de données demandées, vocabulaire employé pour effectuer la demande, tonalité de la sollicitation, modalités de réponse proposées à l'internaute, possibilité offerte au consommateur de ne plus être sollicité ultérieurement). Pour les entreprises, il convient donc de mobiliser avec pertinence ces dimensions contextuelles dans le but d'accrô̂tre l'impact des bénéfices

\footnotetext{
${ }^{9}$ Pour une revue de littérature détaillée sur la variable contextuelle et ses différentes composantes, voir Lemoine (1998).
} 
associés au comportement de dévoilement des internautes ou de réduire l'influence des coûts qui y sont liés.

\section{Discussion des résultats}

Comment faire accepter aux internautes de divulguer leurs données personnelles ? Pour répondre à cette interrogation, 30 entretiens semi-directifs ont été menés auprès d'internautes français. Les résultats obtenus présentent à la fois des intérêts managériaux et théoriques. Sur le plan pratique, tout d'abord, il ressort de nos données :

- que les internautes font preuve d'une très grande lucidité quant aux objectifs commerciaux poursuivis par les entreprises souhaitant collecter les données personnelles de leurs clients (ou de leurs visiteurs);

- que les craintes associées à la divulgation d'informations personnelles sont diverses : peur d'être confronté à des demandes d'informations trop intimes, de ne pas maîtriser le processus de traitement et d'exploitation des données communiquées et peur d'apparaître dans des fichiers. Si la littérature a déjà recensé les trois premiers types de craintes, la quatrième, en revanche, apparaît comme une nouveauté qu'il peut être dorénavant utile à prendre en compte dans l'analyse du comportement de dévoilement des internautes.

Si ces craintes apparaissent, à première lecture, comme rédhibitoires à la transmission d'informations, la réalité est beaucoup plus nuancée. Plus précisément, l'analyse de nos données nous permet de dégager quatre types de stratégies que les entreprises peuvent mettre en œuvre afin de contourner les motifs de non divulgation des données personnelles des internautes :

- offrir une contrepartie aux internautes (sous forme d'avantages monétaires ou de services gratuits offerts);

- faire preuve de pédagogie dans la présentation de la demande d'information (en spécifiant notamment son utilité et sa finalité) ;

- soigner la formulation de la demande (en veillant à ce que la requête ne porte que sur un nombre limité de données, en soignant la tonalité de la demande qui ne doit pas apparaître comme agressive/insistante, en mobilisant un vocabulaire compréhensible des consommateurs, en offrant aux internautes une liberté de réponse et la possibilité de ne plus être sollicité ultérieurement) ; 
- gagner la confiance des répondants (en prenant soin de décliner précisément l'identité du demandeur, en insistant sur le caractère confidentiel du dévoilement et sur la sécurisation des sites sur lesquels sont collectées les informations).

D’une manière générale, la mise à jour des stratégies prenant en compte les différentes dimensions du contexte de sollicitation des internautes («faire preuve de pédagogie dans la présentation de la demande » et «soigner la formulation de la demande ») constitue le principal apport managérial de cette recherche. En effet, si les entreprises sont coutumières avec le fait de proposer des contreparties aux internautes et/ou de chercher à gagner leur confiance, il semble qu'elles le sont beaucoup moins avec la manière dont elles doivent présenter le contexte dans lequel s'inscrivent leurs demandes d'information (Lemoine, 2001a, 2001b). Notre recherche les renseigne sur de nouveaux leviers d'action qu'elles n'utilisent pas encore mais qui sont susceptibles de favoriser la divulgation des données personnelles des internautes (explicitation de l'utilité et de la finalité de la collecte, type de vocabulaire à employer (ou à éviter), tonalité de la demande, modalités de réponses offertes, nombre d'informations demandées lors de chaque sollicitation).

Sur le plan théorique, cette étude est l'occasion de compléter la littérature existante consacrée à l'analyse et à la compréhension de la relation entre préoccupation pour le respect de la vie privée et dévoilement de soi. Plus précisément, notre recherche vient enrichir la théorie du privacy calculus en mettant à jour de nouvelles variables, en l'occurrence celles liées au contexte de sollicitation des internautes, susceptibles d'améliorer la compréhension du calcul de la valeur perçue de l'échange. Jusqu'à présent, il était précisé dans la littérature que les internautes décidaient de fournir (ou non) leurs données personnelles sur la base du calcul de la valeur perçue de l'échange correspondant au différentiel entre les bénéfices et les coûts associés à la divulgation des données (Dinev et Hart, 2006). Si notre étude a bien mis en évidence ces différents bénéfices (contreparties financières, offre de produits et/ou de services) et coûts (les motifs de non divulgation des données personnelles), elle a également permis d'attirer notre attention sur sept nouvelles composantes du contexte de sollicitation des internautes susceptibles de modérer leur importance respective dans la détermination de la valeur perçue de l'échange (figure 1). 
Figure 1 : Modèle d'évaluation de la valeur d'échange

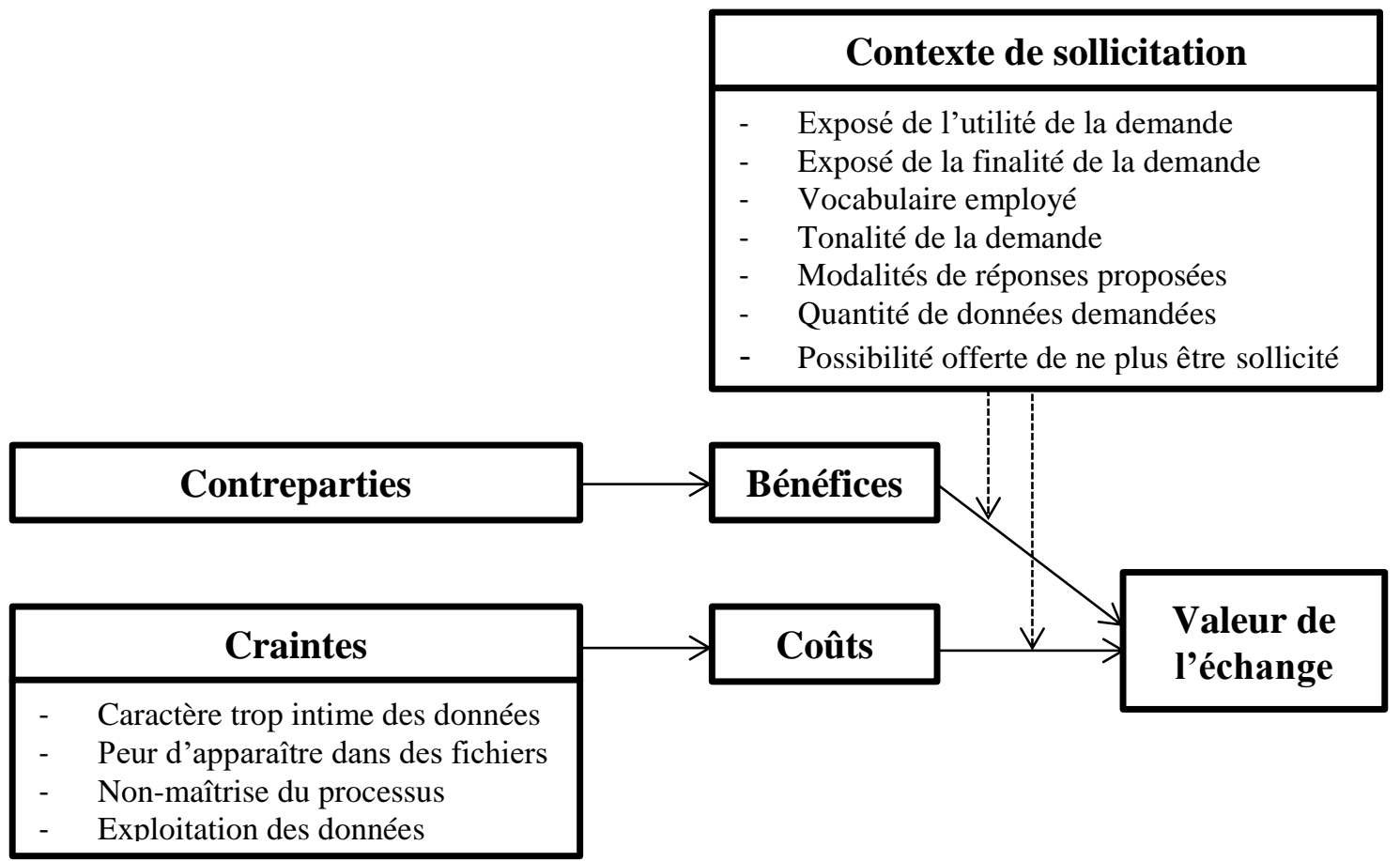

Les verbatim qui suivent illustrent l'impact de ces variables contextuelles sur le comportement de dévoilement des internautes :

«S'ils me proposent une contrepartie, je suis prêt à leur communiquer mes données personnelles...et ce d'autant plus qu'ils me le demandent de manière claire et de façon bienveillante » (Homme, 34 ans, plusieurs fois par semaine) ;

«Ca me dérange de donner si je ne bénéficie pas en retour de promos...et encore plus si c'est demandé de manière trop insistante...trop agressive » (Femme, 19 ans, plusieurs fois par jour);

«Si leurs questions me semblent aller trop loin, ça risque de me gêner...à moins qu'ils prennent le temps de m'expliquer l'objectif de leur demande » (Femme, 64 ans, plusieurs fois par mois) ;

«Je risque de ne pas répondre s'ils m'envoient ensuite des tas de choses que je $n$ 'ai pas demandé.......... 'est différent s'ils s'engagent à ne pas le faire » (Homme, 33 ans, plusieurs fois par semaine).

Notre étude a donc permis d'enrichir le cadre théorique du privacy calculus en permettant de mieux comprendre :

- comment l'internaute évalue la valeur perçue de l'échange et décide, en conséquence, de divulguer ou non ses données personnelles. Cette valeur dépend directement des bénéfices et des coûts recensés qui, à leur tour, sont 
fonction de la perception des finalités de la collecte et des motifs de non divulgation des données. Ainsi, plus l'internaute identifie les finalités de la collecte de ses informations personnelles, plus on peut supposer que ses attentes en termes de bénéfices à recevoir seront importantes. De même, plus ses motifs de non-divulgation sont prégnants, plus ils constituent des coûts élevés susceptibles de réduire la valeur de l'échange et donc la fourniture de ses données ;

- comment les contextes de sollicitation des internautes viennent modérer les relations entre, d'une part, les bénéfices et la valeur de l'échange et, d'autre part, entre les coûts et cette dernière.

Si cette étude permet de dégager des contributions à la fois managériales et théoriques, il convient néanmoins de rappeler qu'elle repose sur une étude qualitative empêchant, de ce fait, toute généralisation des résultats présentés. Pour y parvenir, il conviendrait d'entreprendre, à l'avenir, une étude quantitative ${ }^{10}$ visant :

- à évaluer l'importance de chaque crainte exprimée dans la décision de ne pas divulguer des données personnelles ;

- à apprécier l'impact de chacune des stratégies recommandées aux entreprises sur la réduction des motifs de non transmission des informations ;

- à associer des profils d'internautes précis (en termes de caractéristiques sociodémographiques, de familiarité et d'expertise avec Internet) à chacun des motifs de non transmission des informations ;

- à confirmer l'effet modérateur de chacune des sept composantes contextuelles de la sollicitation entre les bénéfices et les coûts associés à la divulgation des données personnelles et le comportement de dévoilement des internautes.

\section{Références bibliographiques}

\footnotetext{
${ }^{10}$ Elle pourrait prendre la forme d'une enquête par questionnaire administrée auprès d'un échantillon représentatif d'internautes français.
} 
Bélanger F. et Crossler R.E. (2011), Privacy in the digital age: a review of information privacy research in information systems, MIS Quarterly, 35, 4, 1017-1041.

Castaneda A.J. et Montoro F.J. (2007), The effect of Internet general privacy concern on customer behaviour, Electronic Commerce Research, 7, 117-141.

Dinev, T. et Hart P. (2006), An extended privacy calculus model for e-commerce transactions, Information Systems Research, 17, 1, 61- 80.

Gauzente C. (2003) Vie privée et internet: la pratique des sites français, Décisions Marketing, 30, 7-16.

Gurau C., Ranchhod A. et Gauzente C. (2003), To legislate or not to legislate : a comparative exploratory study of privacy-personalisation factors affecting French, UK and US web sites, Journal of Consumer Marketing, 20, 7, 652-654.

Hui K.L., Teo H.H et Sang-Yong T.L. (2007), The value of privacy assurance: an exploratory field experiment, MIS Quarterly, 31, 1, 19-33.

Kaplan D. (2010), Informatique, Libertés, Identités, Paris: Pearson, Fip Editions.

Lancelot Miltgen C. (2008), Propension à fournir des données personnelles mensongères sur Internet : une étude exploratoire, Systèmes d'Information et Management, 13, 3, 1-34.

Lancelot Miltgen C. (2010), Dévoilement de données personnelles et contreparties attendues: une approche typologique et interculturelle, Systèmes d'Information et Management, 15, 4, 45-91.

Lusoli W. et Miltgen C. (2009), Young people and emerging digital services: an exploratory survey on motivations, perceptions and acceptance of risks, JRC Scientific 
and Technical Reports EUR 23765 EN, W. Lusoli, R. Compañó \& I. Maghiros (Eds.), Sevilla: EC JRC IPTS,

Lemoine J.F. (1998), Le rôle du projet de consommation dans le choix du produit, Revue Française de Gestion, 121, 53-64.

Lemoine J.F. (2001a), Comment tenir compte des émotions du consommateur, Revue Française de Gestion, 134, 47-60.

Lemoine J.F. (2001b), Contextes d'achat et critères de choix : acheter pour soi ou pour les autres », Décisions Marketing, 22, 25-31.

Laufer R. et Wolfe M. (1977), Privacy as a concept and a social issue: a multidimensional developmental theory, Journal of Social Issues, 33, 22-42

Li Y. (2011), Empirical studies on online information privacy concerns: literature review and an integrative framework, Communications of the Association for Information Systems, 28, 1, 453-496.

Lwin M. et Williams J. (2003), A model integrating the multidimensional developmental theory of privacy and theory of planned behavior to examine fabrication of information online, Marketing Letters, 14, 4, 257-272.

O’Connor P. (2003), What happens to my information if I make a hotel booking online: an analysis of online privacy policy use, content and compliance by the international hotel companies, Journal of Services Research, 3, 2, 5-28.

Paine C., Reips U.D., Stieger S., Joinson A., et Buchanan T. (2007), Internet users' perceptions of privacy concerns' and privacy actions, International Journal of HumanComputer Studies, 65, 6, 526-536.

Pavlou P.A. (2011), State of the information privacy literature: where are we now and where should we go ?, MIS Quarterly, 35, 4, 977-988. 
Phelps J., Nowak G. et Ferrell E. (2000), Privacy concerns and consumer willingness to provide personal Information, Journal of Public Policy and Marketing, 19, 1, 27-41.

Posey C., Lowry P.B., Roberts T.L. et Ellis T.S. (2010), Proposing the online community self-disclosure model: the case of working professionals in France and the U.K. who use online communities, European Journal of Information Systems, 19, 181-195.

Smith H.J., Dinev T. et Xu H. (2011), Information privacy research: an interdisciplinary review, MIS Quarterly, 35, 4, 989-1015.

Solove D. (2006), A taxonomy of privacy, University of Pennsylvania Law Review, 154, $3,477-560$.

Stone E. et Stone D. (1990), Privacy in organizations: theoretical issues, research findings and protection mechanisms, Research in Personnel and Human Resources Management, $8,349-411$.

Ward S., Bridges K. et Chitty B. (2005), Do incentive matter? An examination of online privacy concerns and willingness to provide personal and financial information, Journal of Marketing Communications, 11, 1, 21-40.

Westin A. (1967), Privacy and Freedom, New York: Atheneum.

Wirtz, J., Lwin M. et Williams J.D. (2007), Causes and consequences of consumer online privacy concern, International Journal of Service Industry Management, 326-348.

Xu H., Luo X., Carroll J.M. et Rosson M.B. (2011), The personalization privacy paradox: an exploratory study of decision making process for location-aware marketing, Decision Support Systems, 51, 42-52. 